\title{
Chapter 10 \\ The Government Responds: The Agency for Healthcare Research and Quality
}

When the IOM report started the patient safety movement by converting the safety interest of a few into the concern of the many, those who wished to enter this emerging field had little to work with: few measures, few proven safe practices, and few standards. For the patient safety movement to blossom in the ways envisioned by the IOM, a substantial amount of foundational work would be necessary. Only the government could provide the resources that were needed to accomplish this work.

Fortunately - amazingly, actually - the federal government was ready and willing to provide those resources, thanks to the recent work by John Eisenberg, director of the Agency for Healthcare Policy and Research (AHCPR) and his team. The Agency had been through a tough patch. Commissioned in 1989 to conduct health services research and develop practice guidelines, it did just that under it first director, Jarrett Clinton, a career PHS bureaucrat. But it lost many of its supporters in Congress in 1994 when the Republicans gained control of both the House and the Senate on a pledge to broadly reduce government: the Contract with America. The federal budget for FY 1996 became the focus of an extraordinarily contentious battle between the administration and Congress.

The Agency was caught in the crosshairs. It was linked to the failed Clinton health reform initiative, to which it had supplied data, and its effort to develop practice guidelines was unpopular with many 
physicians. Worse, its guidelines program had been criticized as being ineffective by the GAO, PPRC, and OTA.

The proverbial "straw" was when the Agency issued a guideline for spine surgery that concluded that there was no evidence to support spinal fusion to treat back pain. Their integrity and pocketbooks challenged, the orthopedists lobbied fiercely to curtail the agency. Enough Congressmen had had back operations or philosophically agreed that the government had no business dictating practice, that they set out to eliminate the agency by reducing its funding to zero.

The House Budget Committee under Rep. John Kasich (R-OH) made AHCPR a symbol of waste and put the agency's name on its "hit list" of 140 discretionary programs to be eliminated [1]. Rep Sam Johnson of Texas mocked it as "the Agency for High Cost Publications and Research." [2] The joint House-Senate committee conference report in June 1996 called for complete elimination of the agency's funding.

By then, Donna Shalala, secretary of Health and Human Services (HHS), had replaced Jarrett Clinton with Cliff Gaus, the highly respected former head of the Association for Health Services Research (AHSR). Gaus went on a campaign to save the Agency. He listened to insurers, hospitals, doctors, and consumers and reframed its agenda to dissemination of guidelines rather than development through a new National Guidelines Clearinghouse to coordinate private sector guidelines.

He partnered with clinical and professional leaders to create the Quality Measures Clearinghouse and enlisted the Health Insurance Association of America (HIAA), the AMA, the Association of American Medical Colleges (AAMC), and the American Association of Health Plans (AAHP) to put their lobbyists to work on Congress for support [3].

The strategy worked, although at a price. In the final appropriations bill, the agency ended up with an appropriation of $\$ 125$ million, a 21 percent cut from FY 1995. The Agency was directed to stop developing practice guidelines, but it survived. By this time, Gaus had had enough. He believed it was time for a physician to direct the Agency. He recommended John Eisenberg to Shalala, noting that in addition to his superb professional qualifications, he was also her personal physician [3]. 
Shalala agreed. She was determined to improve quality of care and realized that patient safety could be the wedge. Interest in safety was rising as the result of our research showing the high rate of preventable harm and the efforts of the AAAS and the NPSF. She thought John was just the person to launch a new effort. And, indeed he was.

Eisenberg brought new stature and an impressive set of strengths to the job. He was a nationally known health services researcher who was chairman of the Department of Medicine and physician in chief at Georgetown University, an IOM member, and a former AHSR president. Having chaired the Physician Payment Review Commission (PPRC) for several years, he had developed trusting relationships with key staff on both sides of the aisle. As a physician he brought increased legitimacy to the agency on matters pertaining to clinical care. He was by consensus both brilliant and politically skilled [3].

For John, it was a dream job. It would enable him to leverage his knowledge and skills on the national stage-if he could get Congressional support. Shalala promised to "have his back." He set to work to rebuild the political stature of the Agency on the Hill, shoring up relationships with HHS and members of Congress on both sides of the aisle. He brought in Gregg Meyer to head up the Center for Quality Measurement and Improvement and Nancy Foster as coordinator.

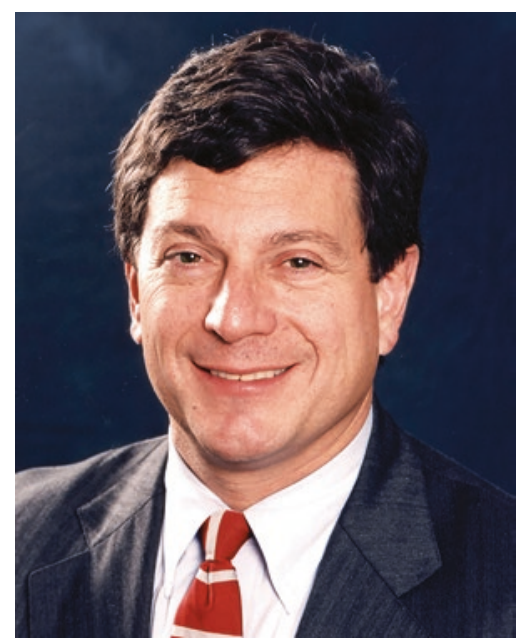

John Eisenberg. (Picture courtesy of the Agency for Healthcare Research and Quality. All rights reserved) 
The battle for survival over, the stage was set for moving ahead. In early 1997, the president had established the Advisory Commission on Consumer Protection and Quality in the Health Care Industry and appointed Shalala and labor secretary Herman as co-chairs [4]. In response to its report in 1998, the president established an umbrella organization to coordinate administration efforts to improve quality: the Quality Interagency Coordination Task Force (QuIC), led by Eisenberg and co-chaired by Shalala and Herman. In addition to AHCPR, QuIC included HHS and the Departments of Labor, Veterans Affairs, Defense, and Commerce [5].

In 1998 the tide in Congress turned when Gingrich left the speakership in disgrace. Gaus' efforts had persuaded Congress of the importance of patient safety and the need for its support. The Agency was working with Congress to change its name and mission before the IOM report came out. As a result, a week later, on December 6, 1999, Congress passed the Healthcare Research and Quality Act of 1999 [6] that amended Title IX of the Public Health Service Act to replace AHCPR with the Agency for Healthcare Research and Quality (AHRQ).

Not only did this act get rid of the hated 10-year-old AHCPR, but it greatly expanded the Agency's role in patient safety, calling on it to "conduct and support research and build private-public partnerships to: 1) identify the causes of preventable health care errors and patient injury in health care delivery, 2) develop, demonstrate, and evaluate strategies for reducing errors and improving patient safety; and 3) disseminate such effective strategies throughout the health care industry." [7] It was an incredibly farsighted mandate and a remarkable boon for the cause. In retrospect, it was extraordinary that Congress would designate an agency to focus on patient safety - and fund it.

\section{Response to the IOM Report}

The IOM report in late 1999 changed everything. Within days of its release, president Clinton called on QuIC to analyze the report and make recommendations. Thanks to the amount of planning that John and his colleagues had done before the report came out, just 60 days later, in early 2000, the Task Force issued its report, Doing What 
Counts for Patient Safety: Federal Actions to Reduce Medical Errors and their Impact, which made more than 100 recommendations for federal entities to address safety issues [8].

Doing What Counts was a blueprint for moving ahead in patient safety. Its recommendations were directed at all government departments. It called on AHRQ to take immediate action to establish the Center for Quality Improvement and Patient Safety (CQuIPS) ${ }^{9}$ and for AHRQ, the Centers for Disease Control (CDC), the Food and Drug Administration (FDA), and VA to cooperate on research on errors, reporting systems, and applied research on patient safety. CQuIPS was called on to build a national system of errors reporting, promote the development and dissemination of evidence-based patient safety practices, and develop patient safety questions for inclusion in the patient experience survey, Consumer Assessment of Healthcare Providers and Systems (CAHPS).

The National Quality Forum (NQF) was asked to define within 12 months a set of egregious errors that are preventable and should never occur. All federal agencies providing healthcare were directed to develop systems to identify and report and learn from errors. The Health Care Financing Agency (HCFA), which paid for Medicare, was called on to promote the use of error-reduction initiatives by healthcare institutions and to require hospitals participating in the Medicare Program to implement medical error reduction programs.

The FDA was to develop standards for proprietary drug names and for packaging and labels to prevent dosing and drug mix-ups. The VA was asked to invest $\$ 47.6$ million to increase patient safety training for staff, and the Department of Defense (DoD) was directed to invest \$64 million in FY 2001 to implement a new computerized medical record system.

The scope was breathtaking. Healthcare had never seen anything like it.

Shalala asked Eisenberg to brief the president on the report. When he did, he found that Clinton had not only read the entire report but understood it. He asked John whether it was possible, as the IOM report challenged, for healthcare to reduce preventable deaths by $50 \%$ in 5 years. John said yes, or even in 2 years or 1 year-because hospitals would manipulate their measures to make it appear that happened. 
Such a requirement would divert their attention from improvement to measures. The president saw the wisdom of his answer, publicly approved the report, and called on all federal health agencies to implement the Task Force recommendations.

Congress did its part and appropriated an additional \$50 million to the Agency for patient safety research. This single act was crucial to building a cadre of researchers to perform the scientific studies needed to advance the field. It would help establish patient safety as an academic discipline with papers published in the leading journals. It did, in fact, have that effect. Within 2 years, 81 grants were awarded and $100 \mathrm{~s}$ of researchers were working on patient safety issues. Without this federal support, research in patient safety would have been slow in coming and spotty at best. It was a foundational initiative.

John Eisenberg was the driving force behind this reinvigorated Agency. His vision of a safer future and how to get there attracted to the Agency an exceptional group of leaders: Gregg Meyer, Carolyn Clancy, and Nancy Foster, among others, who were motivated to get these programs going. Clancy had directed the Outcomes Center at AHCPR prior to the change. They and others who worked at or with AHRQ, or were funded by it, became the first generation of people who made safety their careers. More than any other person, John Eisenberg helped establish patient safety as a science, as a practice, and as an imperative.

Tragically, John could not see the effort through. He died from a brain tumor in early 2002, just as his efforts were beginning to pay off. To honor John's memory, the NQF and The Joint Commission established the John M. Eisenberg annual awards in patient safety that recognize exceptional contributions by individuals and organizations to the advance of patient safety.

Carolyn Clancy took over as director and expanded AHRQ's activities and influence as the major force advancing patient safety. She put emphasis on working strategically with multiple stakeholders-hospitals, health plans, federal and private systems, and patients and families - to actually make changes to make healthcare safer. Patient advocates were added to the National Advisory Council. The CAHPS Hospital Survey (HCAHPS) was launched in collaboration with the Centers for Medicare and Medicaid Services (CMS). Proposals were 
made to link malpractice reforms with improvement in disclosure, apology, and compensation.

The breadth and depth of AHRQ activities in patient safety are awesome. In addition to funding research, it carries out evaluations of evidence, develops and standardizes safety measures and indicators, develops and maintains surveys, trains patient safety specialists, educates the profession and the public, and provides tools for healthcare organizations to improve safety.

AHRQ has played a critical role in establishing the field of patient safety. A full description of all of AHRQ's programs would require several volumes. What follows is a brief summary of its initial programs in patient safety. Much of this information comes from the AHRQ website, ahrq.gov, and AHRQ's 10-year report, Advancing Patient Safety: A Decade of Evidence, Design and Implementation [7], also on the AHRQ website.

\section{AHRQ Programs}

\section{The Center for Quality Improvement and Patient Safety} (CQuIPS) In 1998, prior to the post-IOM reorganization, AHCPR created a center for quality improvement to bring together agencies within HHS to collaborate on improving quality of care and coordinate support for research. Eisenberg expanded this to include patient safety, renamed it, and brought in Gregg Meyer to organize it. Meyer hired Jim Battles to run the research grants program.

CQuIPS supports investigator-initiated research on patient safety, measurement, and reporting. It "develops and disseminates reports and information on health care quality measurement, reporting, and improvement, collaborates with stakeholders ... to implement evidence-based practices, accelerating and amplifying improvements in quality and safety for patients." [9] CQuIPS is also responsible for CAHPS and WebM\&M (see below). The Center has been the clearly identifiable part of government devoted to patient safety.

Consumer Assessment of Healthcare Providers and Systems (CAHPS) This survey initiative began in 1995, before the Agency 
became involved in patient safety, in response to the recognition that quality of care issues that are important to consumers, such as communication skills of providers and ease of access to healthcare, were often overlooked. The obvious way to find out about them was to ask patients. The Agency began to fund, oversee, and work closely with a consortium of research organizations to conduct research on patient experience and develop the survey.

The survey has since been expanded to ask patients to evaluate their experiences with health plans, providers, and healthcare facilities regarding care coordination, shared decision-making, and patient engagement. The survey is now widely used by healthcare organizations, health plans, purchasers, consumer groups, and accreditation organizations to evaluate providers and improve quality and safety of care. It has been a major factor in teaching clinicians and hospitals to be more aware of patient's concerns and to engage them more meaningfully in their care. It has magnified their voice.

Patient Safety Indicators (PSI) What everyone wants-CMS, policy-makers, hospitals, and the public - is an overall measure of safety. How bad is it? Are we getting better? But there was no such measure, nor even a set of standardized measures that the nation or a healthcare organization could use to identify its safety problems. However, CMS and other payers required hospitals to use ICD-9 billing codes for specific harmful events, such as infections, pressure ulcers, surgical complications, falls, CLABSI rates, etc. Why couldn't hospitals use this "administrative" data to assess and improve their performance?

Under Gregg Meyer's direction, AHRQ created a list of 20 patient safety indicators using billing codes. To develop an overall score, they calculated a weight for each based on national data on risk, reliability of the measure, and extent of harm. The hospital could monitor the rate of each indicator, multiply it by its weight, and sum the values to get an overall measure of safety in the institution. While incompleteall safety risks were not measured-it was better than nothing and would help hospitals know where to focus their safety efforts.

When the PSIs were released in 2001, AHRQ made it clear that the indicators were designed only for hospitals' internal use for improvement: to "provide information on potentially avoidable safety 
events ...that can be used to help hospitals assess the incidence of adverse events and identify issues that might need further study." [7]

It was not to be. Despite these caveats, CMS and other payers began to use them not only to assess hospitals performance, but to reduce payments if they were deficient. Suddenly PSIs were viewed not as tools for improvement, but as instruments of punishment-a complete inversion of what we were trying to do in patient safety.

The patient safety community was appalled. Hospitals quickly directed their efforts away from improvement to coding of claims, "gaming" the data to minimize penalties. This was exactly what Eisenberg had told the president would happen if hospitals were required to reduce preventable mortality by $50 \%$ in 5 years. In the end PSIs may have done more harm than good over the years. Meyer agrees. He considers them "the worst thing I ever did" at AHRQ.

Evidence-Based Practices Even in these early days, there were a number of established safe practices available. Which should healthcare organizations use? Which safe practices were effective? The newly established standard setter, the National Quality Forum, needed to know, so it turned to AHRQ. The Agency commissioned an Evidence-Based Practices group at the University of California at San Francisco (UCSF), led by Bob Wachter and Kaveh Shojania, to review the evidence and report in 6 months.

Many of us were looking forward to the report, so when it appeared, it was a shocker to find that only a small number of practices were found to have evidence of effectiveness, and most of those with the highest ratings were rarely used or fairly esoteric. These practices received high marks because someone had done a randomized controlled trial (RCT) of their effectiveness. Most of the safe practices in widespread use were not on the list because they had not been subjected to RCTs. The UCSF group had done what they were asked to do: follow the evidence. However, the evidence came from studies that individuals did because of their interest in a specific practice, not because it prevented many errors or was in widespread use [10].

David Bates, Don Berwick, and I were concerned that this would send the wrong message that practices without evidence should be abandoned, so we crafted a critique that was published in JAMA: What practices will most improve safety? Evidence-based medicine 
meets patient safety [11]. JAMA published it together with the authors rebuttal: Safe but sound: patient safety meets evidence-based medicine [12] as part of a point-counterpoint analysis to help readers understand the types of evidence needed to support use of a new practice.

Our major point was that the many accepted safe practices in current use should not be abandoned just because they had never been subjected to a controlled trial, as required in the review. (The standing joke about RCTs is that no one had ever done a randomized trial of the effectiveness of parachutes!) Handwashing, read-back, site marking, and unit dosing, for example, were practices without evidence that were clearly of value and should not be abandoned.

The paper was written both to reassure those working on the "front lines" of safety in hospitals and to serve as a resource for the NQF Safe Practices Steering Committee. It seems to have been of some value to both. The Safe Practices criteria were expanded to include experiential evidence of effectiveness. The final NQF list included 34 approved safe practices [13].

WebM\&M To engage and inform physicians, AHRQ initiated WebM\&M, using the familiar format of mortality and morbidity rounds to make available analysis of real-world medical error cases by experts, monthly. Edited by Bob Wachter, founder of the hospitalist specialty and later chair of the American Board of Internal Medicine, about a third of the cases are also developed as Spotlight Cases that are interactive learning modules for CME. This has proven to be one of the agencies most popular offerings.

Survey on Patient Safety Culture (SOPS) To support the development of a culture of patient safety, AHRQ sponsored the development of patient safety culture assessment tools for hospitals, nursing homes, ambulatory outpatient medical offices, community pharmacies, and ambulatory surgery centers. These surveys enable healthcare organizations to assess staff perceptions of various aspects of patient safety culture. They have played an important role in creating a culture of safety.

National Healthcare Quality and Disparities Report To justify funding, Congress wants to see results. Part of its 1999 mandate was 

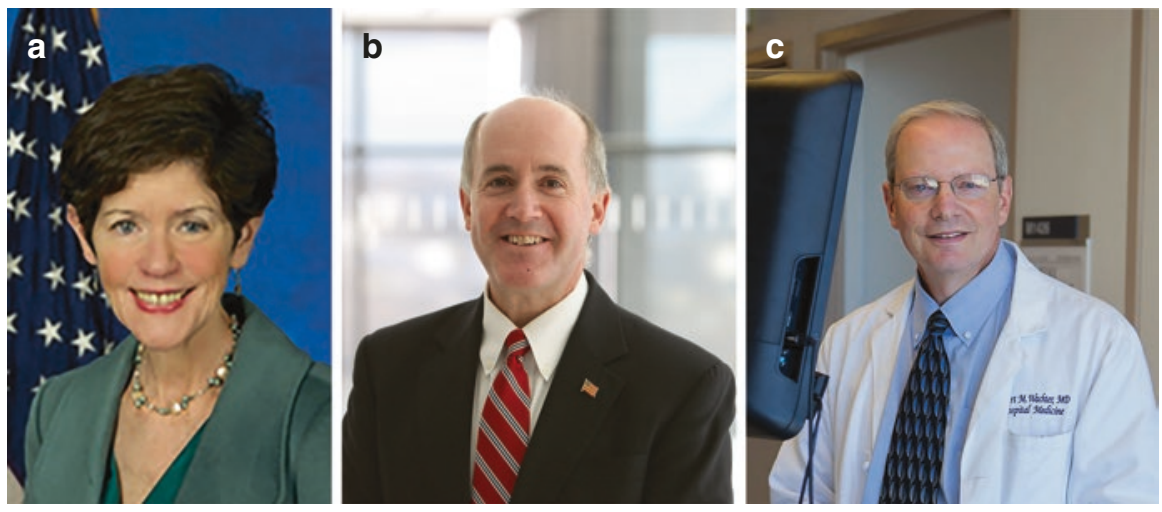

(a) Carolyn Clancy, (b) Gregg Meyer, and (c) Bob Wachter. (All rights reserved)

that AHRQ produce annual reports on healthcare quality and disparities. The quality reports present trends for access to care, affordable care, care coordination, effective treatment, healthy living, patient safety, and person-centered care. The disparities report provides comparative information according to race and ethnicity, income, and social determinants of health. These reports have helped keep patient safety on the agenda and motivate other agencies to work on safety.

Education and Training AHRQ has developed educational programs for practitioners in several areas. TeamSTEPPS® is a training program based on an evidence-based set of ready-to-use materials and curriculum to improve teamwork in healthcare organizations by teaching communication and teamwork skills. The Patient Safety Improvement Corps (PSIC) is a partnership with the Department of Veterans Affairs to train midlevel professionals in investigation of medical errors and initiating improvements.

Advancing Pharmacy Health Literacy Practices Through Quality Improvement is a set of modules to help pharmacy faculty integrate health literacy and health literacy quality improvement into the education of pharmacy students and residents.

Central line-associated bloodstream infections (CLABSI) were a serious cause of preventable injury and death. CLABSI Tools help care units implement evidence-based practices to eliminate central lineassociated bloodstream infections. The Comprehensive Unit-based 
Safety Program (CUSP) toolkit developed by Peter Pronovost's team includes training tools to make care safer by improving the foundation of how physicians, nurses, and other clinical team members work together [14].

Several of these education and training programs have had substantial impact. CLABSI Tools were used with the CUSP toolkit in a highly successful nationwide initiative led by Pronovost that dramatically reduced CLABSI rates in more than 1000 hospitals across the country [15]. The Patient Safety Improvement Corps (PSIC) has trained teams in every state and has been a major force in disseminating patient safety knowledge throughout the country. TeamSTEPPS has trained 1000 master trainers who in turn train colleagues at their organizations. Almost every hospital now has a patient safety officer; many were trained in this program.

Patient Education AHRQ has published a number of guides for consumers, such as guides for what to do after leaving the hospital, use of blood thinners, diagnosis and treatment, and questions to ask your doctor.

Health Information Technology Suddenly, after only 3 years of supporting the full range of patient safety research, in 2003 Congress directed that AHRQ's \$50 million annual research funding be devoted to research in information technology. This was a shock because the unrestricted funding had been a powerful incentive for developing new knowledge and attracting new investigators to the field. Research is the coin of the realm in academia, and research gave our new field academic respectability. Our researchers' papers were being published and they were being promoted.

But the need for support of health IT was clear. Hospitals and doctors were being required to implement electronic health records (EHRs) and were having serious problems. The funding resulted in hundreds of projects related to all aspects of implementation of health IT, and the staff made sure other projects were funded as well. In 2004, AHRQ provided \$139 million for more than 100 multi-year demonstration grants and contracts to promote the use of health information technology. 
Patient Safety Network To enable healthcare providers and researchers, as well as administrators and patients, to keep up with and easily access the increasing "firehose" of data and patient safety information, AHRQ created a website, psnet.ahrq.gov, with the latest news, research, legislation, and tools for patient safety.

Healthcare-Associated Infections Network In 2008 Congress directed AHRQ to work with CDC and CMS to develop an action plan to reduce hospital-acquired infections (HAI). Several nonprofit organizations joined what became a major national effort and one of the most successful patient safety campaigns. The reductions in HAI as a result of this program account for a significant share of safety improvement over the past 10 years.

Patient Safety Organizations From the beginning of the patient safety movement, one of the goals has been to develop national or regional reporting systems so hospitals could share medical error information and learn from each other's mistakes. The IOM called for development of these voluntary systems, but it didn't happen, in part because of hospitals' fear that the information would be legally discoverable and used to sue hospitals and doctors for malpractice.

To eliminate this liability and facilitate sharing of patient error data among hospitals, in 2005 Congress established Patient Safety Organizations (PSOs). Medical error information reported to PSO confidentially is protected from legal discovery. AHRQ also coordinated the development of common definitions and reporting formats to standardize data collection. Since the law was passed, a number of PSOs have developed across the country. Their effectiveness varies, but some have been useful vehicles for sharing lessons learned.

\section{Impact of AHRQ Programs}

As is obvious from the above, AHRQ has played an immense role in the development of all aspects of patient safety. It has given substance to "it's not bad people, it's bad systems"-in research, in practice, and in policy. It has been the main funder of patient safety research. It was 
the prime mover developing a cadre of patient safety researchers and training hundreds of patient safety officers.

It furthered the development of measures and set standards for their use. It developed key surveys and large databases that provide the information upon which public policy and private improvement depend. It motivated other federal agencies, such as CMS and CDC, to become major players supporting patient safety.

To evaluate its national patient safety initiative in September 2002, AHRQ entered into a 4-year contract with the RAND Corporation. In 2005 RAND published the first report [16]. It complimented the agency on "an impressive job in starting the patient safety initiative" that balanced research and translational and practice improvements. It specifically commended AHRQ for its work in support of epidemiology research, development of effective practices and tools, building infrastructure, and achieving broader adoption of effective practices.

Given its crucial role-and incredible success - it is disturbing and puzzling that funding for AHRQ has always been somewhat precarious. The support for research has never been adequate: large numbers of excellent proposals go unfunded each year. The sudden shift of research funding to IT mandated by Congress in the early years has been followed periodically by other requirements to target its efforts to areas of Congressional interest at the time.

As Gray observed, AHRQ's political problems are three-dimensional. Congress is willing to support basic research, as it does with $\mathrm{NIH}$, only if it believes the long-term result will be new ways of preventing or treating disease. AHRQ's results so far have apparently not been sufficiently convincing. Second, when the Agency produces work that affects healthcare practice or policy, it attracts enemies who are vested in the status quo (recall the orthopedists and spine surgery). Third, the agency's work is significant to many parties: policy-makers, decision-makers (providers, purchasers, patients), and researchers. They understandably have competing ideas about how the agency's limited resources should be spent [3].

Thus, despite appeals from many health policy experts over the years to "billionize" the Agency, annual funding has remained in the $\$ 300-500$ million range. Funding was gradually increased during the Obama years but then cut back by the current administration, which also discontinued several programs. 
To health policy experts, it seems obvious that AHRQ should become an institute as part of $\mathrm{NIH}$, with annual funding at \$1-2 billion level, which is less than that currently provided for several institutes for conditions that affect far fewer people and cause far fewer deaths. Surely, it is as important to fund research on how we deliver care as it is to fund research on what care we deliver. NIH has always enjoyed broad bipartisan and public support. If there were a National Institute for Quality and Safety, funding for patient safety would be adequate and secure. A reasonable hope.

\section{References}

1. U.S. House Committee on the Budget. Discretionary spending and control act of 1995. Washington, D.C: U.S. G.P.O; 1995. p. 1-64.

2. Sequential votes postponed in committee of the whole. Washington, D.C. Congressional Record; 1995. H8413.

3. Gray BH, Gusmano MK, Collins SR. AHCPR and the changing politics of health services research. Health Affairs. 2003;22:W3-283-307.

4. President clinton announces advisory commission on consumer protection and quality in the health care industry. Washington, D.C.: The White House Office of the Press Secretary; 1997.

5. Clinton WJ. Memorandum on establishment of the quality interagency coordination task force. Washington, D.C: The White House Office of the Press Secretary; 1998.

6. 113 Stat. 1653 - Healthcare Research and Quality Act of 1999. S580, Public Law 106-129. Washington, D.C.: U.S. Government Printing Office; 1999:1653-1676.

7. Agency of Healthcare Research and Quality. Advancing patient safety: a decade of evidence, design and implementation 2009 November 2009.

8. Quality Interagency Coordination Task Force. Doing what counts for patient safety: federal actions to reduce medical errors and their impact 2000 February 2000.

9. Center for Quality Improvement and Patient Safety (CQuIPS). Agency for healthcare research and quality, 2012 at https://www.ahrq.gov/cpi/centers/ cquips/index.html.

10.Shojania K, Duncan B, McDonald K, Markowitz A, editors. Making health care safer: a critical analysis of patient safety practices. Rockville: Agency for Healthcare Research and Quality; 2001.

11.Leape LL, Berwick DM, Bates DW. What practices will most improve safety? Evidence-based medicine meets patient safety. JAMA. 2002;288:501-7. 
12. Shojania KG, Duncan BW, McDonald KM, Wachter RM. Safe but sound: patient safety meets evidence-based medicine. JAMA. 2002;288:508-13.

13. National Quality Forum. Safe practices for better health care: a consensus report. Washington, DC: NQF; 2003. Report No.: NQFCR-05-03.

14.Pronovost PJ, Marsteller JA, Goeschel CA. Preventing bloodstream infections: a measurable national success story in quality improvement. Health Aff. 2011;30:628-34.

15.Eliminating CLABSI, A national patient safety imperative. Final Report on the National on the CUSP: Stop BSI Project. Agency for Healthcare Research and Quality; October 2012.

16. Farley DO, Morton SC, Damberg CL, et al. Assessment of the national patient safety initiative: context and baseline evaluation report I. RAND Health: Santa Monica; 2005.

Open Access This chapter is licensed under the terms of the Creative Commons Attribution 4.0 International License (http://creativecommons.org/ licenses/by/4.0/), which permits use, sharing, adaptation, distribution and reproduction in any medium or format, as long as you give appropriate credit to the original author(s) and the source, provide a link to the Creative Commons license and indicate if changes were made.

The images or other third party material in this chapter are included in the chapter's Creative Commons license, unless indicated otherwise in a credit line to the material. If material is not included in the chapter's Creative Commons license and your intended use is not permitted by statutory regulation or exceeds the permitted use, you will need to obtain permission directly from the copyright holder.

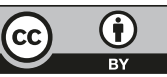

